\title{
Surveillance and response systems for elimination of tropical diseases: summary of a thematic series in Infectious Diseases of Poverty
}

Xia Zhou ${ }^{1,2}$, Peiling Yap ${ }^{3,4}$, Marcel Tanner ${ }^{3,4}$, Robert Bergquist ${ }^{5}$, Jürg Utzinger ${ }^{3,4}$ and Xiao-Nong Zhou ${ }^{2,6^{*}}$

\begin{abstract}
The peer-reviewed journal Infectious Diseases of Poverty provides a new platform to engage with, and disseminate in an open-access format, science outside traditional disciplinary boundaries. The current piece reviews a thematic series on surveillance-response systems for elimination of tropical diseases. Overall, 22 contributions covering a broad array of diseases are featured - i.e. clonorchiasis, dengue, hepatitis, human immunodeficiency virus/acquired immune deficiency syndrome (HIV/AIDS), H7N9 avian influenza, lymphatic filariasis, malaria, Middle East respiratory syndrome (MERS), rabies, schistosomiasis and tuberculosis (TB). There are five scoping reviews, a commentary, a letter to the editor, an opinion piece and an editorial pertaining to the theme "Elimination of tropical disease through surveillance and response". The remaining 13 articles are original contributions mainly covering (i) drug resistance; (ii) innovation and validation in the field of mathematical modelling; (iii) elimination of infectious diseases; and (iv) social media reports on disease outbreak notifications released by national health authorities. Analysis of the authors' affiliations reveals that scientists from the People's Republic of China (P.R. China) are prominently represented. Possible explanations include the fact that the 2012 and 2014 international conferences pertaining to surveillance-response mechanisms were both hosted by the National Institute of Parasitic Diseases (NIPD) in Shanghai, coupled with P.R. China's growing importance with regard to the control of infectious diseases. Within 4 to 22 months of publication, three of the 22 contributions were viewed more than 10000 times each. With sustained efforts focusing on relevant and strategic information towards control and elimination of infectious diseases, Infectious Diseases of Poverty has become a leading journal in the field of surveillance and response systems in infectious diseases and beyond.
\end{abstract}

Keywords: Infectious diseases, Tropical diseases, Health systems, Surveillance and response systems, Elimination, People's Republic of China

\section{Multilingual abstracts}

Please see Additional file 1 for translations of the abstract into the six official working languages of the United Nations.

\footnotetext{
* Correspondence: ipdzhouxn@sh163.net

${ }^{2}$ National Institute of Parasitic Diseases, Chinese Center for Disease Control and Prevention, 207 Rui Jin Er Road, Shanghai 200025, People's Republic of China

${ }^{6}$ Key Laboratory on Parasite and Vector Biology, Ministry of Health, WHO Collaborating Centre for Topical Diseases, 207 Rui Jin Er Road, Shanghai 200025, People's Republic of China

Full list of author information is available at the end of the article
}

\section{Background}

Tropical diseases are inextricably linked to populations living in resource-constrained settings $[1,2]$. Infectious diseases of poverty, a collective term coined for infections known to be particularly prevalent amongst marginalized communities, is increasingly used to describe tropical diseases with special transmission routes, such as those that depend on a vector (e.g. malaria; vector is a mosquito) or an intermediate host (e.g. schistosomiasis; intermediate host is a snail) $[3,4]$.

The vision of the BioMed Central (BMC) open-access journal Infectious Diseases of Poverty is in line with the

\section{Biomed Central}


objectives put forth by the World Health Organization (WHO) in its 2012 Global Report [5]. The journal was launched in October 2012 to explore new avenues in research to deepen the understanding of the relationship between infectious diseases and poverty, to contribute to priority-setting and translation of key findings into policies and programmes to control and eliminate these diseases. The expanded "One World - One Health" concept, first proposed by the Wildlife Conservation Society and supported by the Food and Agriculture Organization (FAO), WHO, the World Bank and the United Nations Children's Fund (UNICEF), recognises that the health of people, animals, and societies is intimately linked, and ultimately dependent, on the resilience of the world's life-supporting ecosystems [6]. Based on this concept, Infectious Diseases of Poverty has published a series of original articles and empirical work with reference to analyses of disease burdens, their distributions and the research that is needed in this area. To raise global awareness of infectious diseases of poverty, the journal has not only published original research articles but also scoping reviews that highlight transdisciplinary advances in the science undertaken. Several collections in different areas and on various topics have been published $[3,7]$.

The most recent collection, entitled "Surveillance and response to infectious diseases of poverty", features 22 articles on topics ranging from tropical diseases to recent outbreaks of the H7N9 avian influenza A and the Middle East respiratory syndrome-coronavirus infection (MERS-CoV) with considerable international impact [8-10]. Eleven diseases are included, i.e. clonorchiasis, dengue, hepatitis, human immunodeficiency virus/acquired immune deficiency syndrome (HIV/AIDS), H7N9 avian influenza, lymphatic filariasis, malaria, MERS, rabies, schistosomiasis and tuberculosis (TB). The purpose of this overview is to summarise and systematise the research and shed new light on the plans for elimination of infectious diseases of poverty put forth in the current collection of the journal.

\section{Article collection}

\section{The categories}

This collection of articles published in Infectious Diseases of Poverty highlights surveillance-response systems that are geared towards the elimination of tropical diseases. It consists of 22 contributions; 13 original research articles, five scoping review, a commentary, a letter to the editor, an opinion piece and an editorial (Additional file 2).

The scoping reviews pertain to surveillance-response systems for elimination of tropical diseases, namely (i) surveillance-response systems as the key to eliminate tropical diseases [11]; (ii) surveillance of antimalarial drug resistance in the People's Republic of China (P.R. China) [12]; (iii) challenges and needs to eliminate rabies in P.R. China
[13]; (iv) a review focusing on TB at the wildlife-livestockhuman interface in Zambia [14]; and (v) epidemiology and interactions of HIV-1 and Schistosoma mansoni in subSaharan Africa [15]. The commentary is linked to the rabies piece and discusses the science of elimination more broadly [16]. In a letter to the editor, research priorities in modelling the transmission risk of H7N9 avian influenza are discussed [17]. The opinion piece emphasises that clonorchiasis in P.R. China must be addressed and tackled in a timely manner [18]. The editorial pertains to the elimination of tropical diseases and highlights the importance of surveillance and response [8].

The 13 original research articles $[10,19-30]$ cover a broad array of topics along the entire value chain from innovation to application. The development and validation of mathematical models are discussed, social media communications arising from notifications of disease outbreaks are introduced and in vitro gene silencing (e.g. of independent phosphoglycerate mutase (iPGM) in Brugia malayi to identify the antifilarial drug targets) are featured. Importantly, one article pertains to health policy and planning with a specific focus on surveillance-response mechanisms (Fig. 1a).

\section{Diseases covered and author constellations}

The 22 contributions focus on 11 different diseases, ranging from single diseases (i.e. clonorchiasis, dengue, hepatitis, HIV/AIDS, H7N9 avian influenza, lymphatic filariasis, malaria, MERS-CoV, rabies, schistosomiasis and $\mathrm{TB}$ ) to co-morbidity with non-communicable diseases (e.g. hypertension and diabetes) or risk of infectious diseases among pregnant women. Several other infectious diseases (e.g. diarrhoea, measles and upper respiratory tract infections) are also addressed (Fig. 1b).

As shown in Fig. 2a, there was a spectrum of author constellations ranging from three single-author contributions [12, 16, 21] to collaborative pieces co-authored by up to 10 researchers [11]. More than three-quarters $(77.3 \%)$ of the articles were written by at least three authors, while the median number of authors per article is four. Among the 19 multi-authored contributions, nine papers have been written by authors affiliated with a single country, while the remaining 10 contributions are composed of authors affiliated with different countries. One of the articles has contributing authors affiliated with four different countries [15] and another one with five different countries or regions [17] (Fig. 2b). Hence, the research pursued in this collection represents work of considerable collaborative nature.

A sum-up with respect to authorship reveals a total of 97 contributing scientists. Most of them contributed to a single piece $(n=90,92.8 \%)$, while the remaining seven authors were engaged with two or more articles, up to a maximum of four contributions $[8,11,17,26]$. 

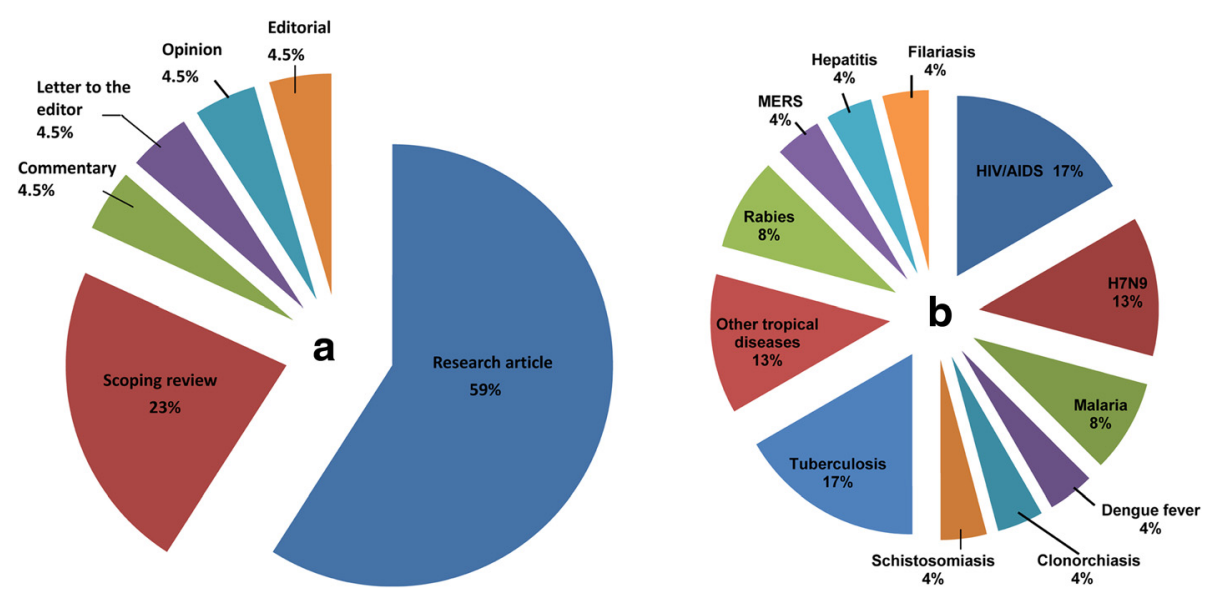

Fig. 1 The categories of the 22 contributions (a) and the main topics discussed (b) in a collection of Infectious Diseases of Poverty pertaining to surveillance-response systems that might lead to elimination of tropical diseases

The provenance of the 22 contributions is depicted in Fig. 3a, with the provenance of the 97 authorcontributions displayed in Fig. 3b. In the latter analysis, each contribution is given the same weight, and hence, the 97 author-contributions account for $100 \%$. Authors with affiliations in two different countries (e.g. P.R. China and South Africa) were accounted for as half for each country. We found that authors affiliated with institutions in P.R. China contributed the highest number of articles $(n=31.8,32.8 \%)$, followed by Nigeria $(n=9.0$, $9.3 \%$ ), the United States of America (USA; $n=8.0$, $8.2 \%)$, Cameroon $(n=8.0,8.2 \%)$ and the United Kingdom (UK; $n=7.3,7.5 \%$ ). Author-contributions from Asia amounted to $41.3 \%$ (40.1/97) followed by authorcontributions from Africa $31.7 \%$ (30.8/97), whilst author-contributions from Europe, North America and South America represent $17.6 \%, 7.4 \%$ and $1.0 \%$, respectively.

In the article-specific analysis, each of the 22 papers was given the same weight. It follows that articles written by a single or only few authors contributed considerably more weight to a given country than articles prepared by several authors from various countries. Taking this weighting into account, P.R. China remains the dominant player (32.8\%), followed by Nigeria (9.3\%) and the USA ( $8.2 \%)$.

\section{Highlights}

\section{Overview}

In their opening overview, Zhou and colleagues [11] provide a comprehensive review on the framework of elimination of tropical diseases by surveillance-response systems. Current strategies and the WHO's roadmap for elimination of neglected tropical diseases (NTDs) [31] are reviewed. A priority research agenda within the "One World - One Health" framework for global health has been developed, including (i) the establishment of a platform for resource-sharing and effective surveillanceresponse systems for Asia Pacific and Africa with an initial focus on efforts aimed at the elimination of lymphatic filariasis, malaria and schistosomiasis; (ii) development of new strategies, tools and approaches, such as improved diagnostics and antimalarial therapy; (iii) rigorous validation
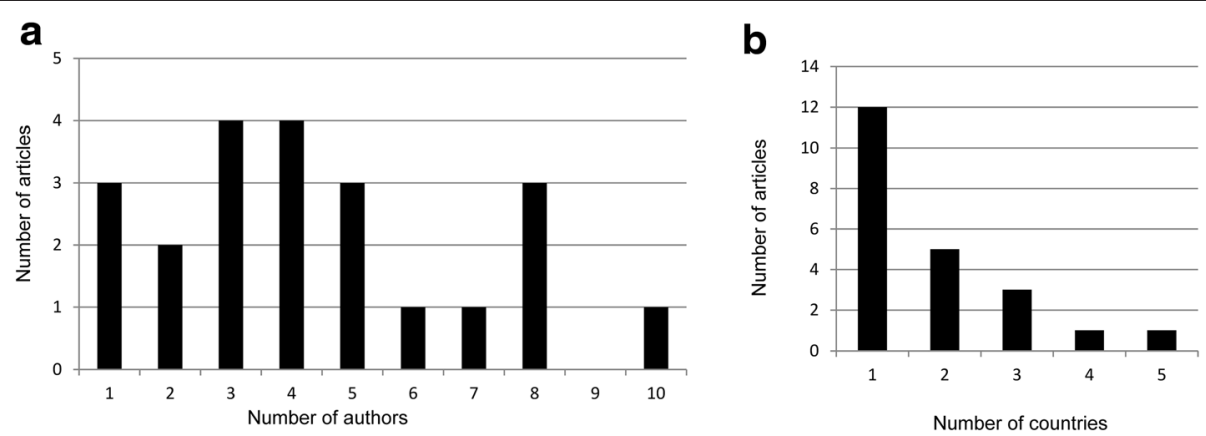

Fig. 2 Histogram showing the number of authors per article (a) and the number of countries the contributing authors are affiliated with (b) in a collection of 22 contributions forming a thematic issue of Infectious Diseases of Poverty pertaining to surveillance-response systems that might lead to elimination of tropical diseases 


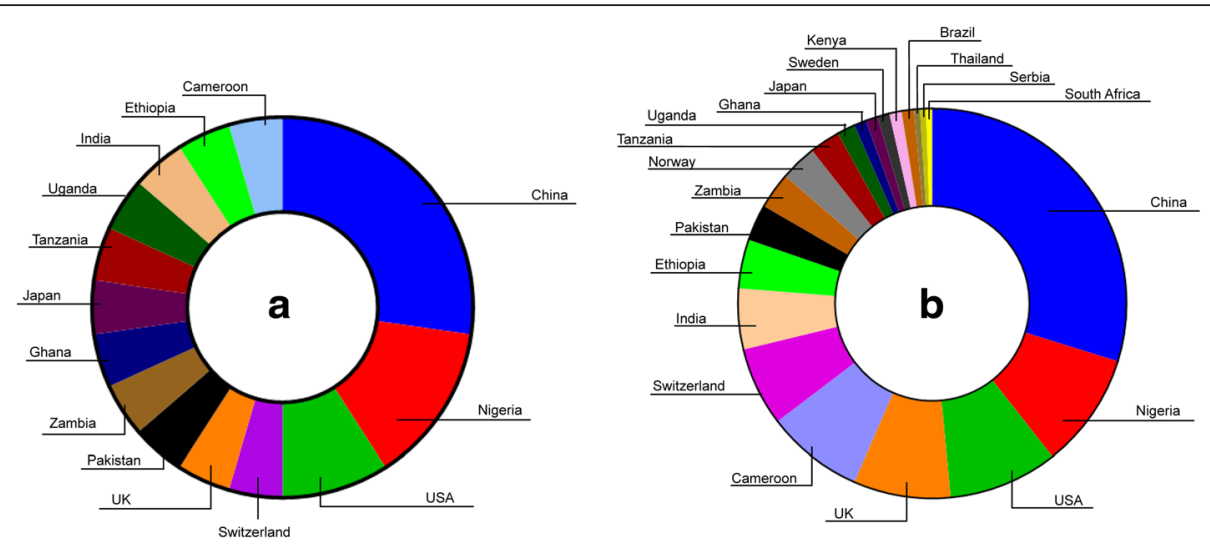

Fig. 3 Provenance of 22 contributions (a) and the 97 author-contributions (b), stratified by authors' country affiliations, in a collection of Infectious Diseases of Poverty pertaining to surveillance-response systems that might lead to elimination of tropical diseases

of surveillance-response systems; and (iv) designing pilot studies for the transfer of Chinese experience of successful surveillance-response systems to endemic countries with limited resources. To emphasise the objectives of this thematic issue based on a "One World - One Health" paradigm, an editorial summarises the outcomes of the First Forum on Surveillance Response Systems Leading to Tropical Diseases Elimination, held in Shanghai in June 2012, including identified research priorities $[3,8,32]$.

\section{Data handling and databases}

Modelling based on a minimum essential database had been proposed in the First Forum on Surveillance Response System Leading to Tropical Disease Elimination and was reiterated in the second forum, held in June 2014, again in Shanghai. A key outcome of the two conferences was the writing-up of the 22 articles featured here. This body of work emphasises the essential role that a minimum essential database can play for effective surveillance and responses that are readily tailored for specific settings.

Data on treatments, co-infections and prevalence of a large variety of tropical diseases are provided in this collection. One scoping review put forth by Liu et al. [12] emphasises that chloroquine-resistant Plasmodium falciparum malaria exists in eight provinces/au tonomous regions of P.R. China. This type of resistance is particularly widespread in the provinces of Hainan and Yunnan. The issue also covers formulations and recommendations of therapeutic regimens for antimalarial drug use in P.R. China for artemisinin/ pyronaridine compounds to promote the rational use of antimalarials and strengthen malaria control/elimination efforts [12].

Contemporary data on immunological, haematological and viral responses and predictors of reported therapeutic failure after initiation of free antiretroviral treatment in
Cameroon were collected to evaluate existing treatmentmonitoring algorithms and to complement efforts to scale-up and improve the management of HIV/AIDS [20].

Since the early 1990s, it has been suggested that HIV and S. mansoni may interact and potentiate the effects of each other within the co-infected human host. A previous collection of Infectious Diseases of Poverty included two articles on the topic of co-infections with one article pertaining to HIV-1/AIDS and S. mansoni that are both widespread in sub-Saharan Africa [15], and the second elucidating on the impact of HIV on TB [30]. The implications of co-infections, both for TB and HIV/AIDS control, are of considerable public health relevance in Ghana, as almost a quarter (23\%) of all TB cases were HIV positive according to a report from 2010. The integration of TB and HIV services has therefore emerged as an essential component of the national response to $\mathrm{TB}$ and $\mathrm{HIV}$ co-infections in this country [30].

Dual burden of disease and other special physical conditions are also covered in this collection, e.g. the report by Amare and colleagues who observed a $6.2 \%$ prevalence of smear-positive pulmonary TB (PTB) in diabetic patients, which turned out to be several-fold higher compared with the general population $(0.39 \%)$. Patients with a previous history of contact with $\mathrm{TB}$ patients, as well as those with prolonged diabetes, were found to be particularly prone to have $\mathrm{PTB}$, making it necessary to screen diabetic patients for PTB infection at follow-up medical visits [27].

Agomo et al. observed that acquired partial immunity against malaria during pregnancy is a key factor in keeping this infection at an asymptomatic level. Depending on the malaria endemicity in a given setting, it is expected that up to $50 \%$ of pregnant women may have parasitaemia, especially in the placenta, although they might not be aware of this [24]. 


\section{Modelling approaches}

A mathematical model for the prediction of risk of infection through needle/syringe-sharing during mass vaccination, including a formula for its calculation, has been developed by Okamoto [21]. Another two research articles with an emphasis on modelling, put forward by Wiwanitkit et al. [17] and Shi et al. [26], discuss the potential risks of H7N9 infection by spatio-temporal characterisation of bird migration and poultry distribution, and estimation of the basic reproduction number for single-strain dengue fever epidemics. The epidemic of H7N9 avian influenza A in the eastern part of P.R. China in early 2013 caused much attention from researchers as well as public health workers, and modelling of its transmission risk resulted in guidelines for active surveillance and control of human influenza infections through intensive intervention in poultry markets. In their article pertaining to dengue transmission by estimating the basic reproduction number for single-strain dengue fever epidemics, researchers from Pakistan did a retrospective analysis of the 2011 epidemic in that region [19].

\section{Application of more sensitive diagnostics}

Use of new technologies supported by mobile or other improved electronic-based approaches and more sensitive diagnostic tools, necessary for settings chosen for elimination, were identified as a key research priority in the First Forum on Surveillance Response System Leading to Tropical Disease Elimination in June 2012 [8]. Nocost social media (Weibo) [10] and mobile phones [25] were employed in two studies to evaluate reactions of the general public with regard to infectious diseases $[10,25]$.

Applications of new molecular diagnostic tests characterised by a high sensitivity, such as the loop-mediated isothermal amplification (LAMP), for real-time diagnosis of malaria infections in a rural or urban high/low transmission setting are recommended in the report of the Second Forum on Surveillance Response System Leading to Tropical Disease Elimination of June 2014 (http:// srs.ipd.org.cn:8080/srs/node/9). Meanwhile, the serological markers for monitoring of Plasmodium vivax transmission in the elimination stage were identified by means of a high throughput analysis [33] to develop more sensitive and convenient diagnostics.

\section{Drug resistance and prevention}

To promote the rational use of antimalarial drugs and strengthen malaria control and elimination, established drug resistance and surveillance of antimalarial drug resistance in P.R. China in the 1980s and 1990s have now been documented, including the formulation of principles and recommendations of therapeutic regimens for the use of antimalarial drugs (artemisinin/pyronaridine compounds) [12]. To identify an antifilarial drug target, in vitro 'gene silencing' of independent phosphoglyceratemutase (iPGM) in the filarial parasite $B$. malayi was performed by Singh et al., whose work suggests that iPGM is essential for both larval and adult stages of $B$. malayi [28].

Dr. William H. Stewart, United States Surgeon General from 1965 to 1969, has been maligned for the statement: "It is time to close the book on infectious diseases, and declare the war against pestilence won". It must be noted, however, that recent investigations fail to find any evidence for him ever having said this [29]. More importantly, in retrospect, emerging new infections and the ever-increasing problems of resistance against available antibiotics clearly shows that now is not the time to close the book on infectious dieases.

\section{Socioeconomics and infectious diseases}

Among the 22 articles pertaining to surveillance-response systems for elimination of tropical diseases, four are associated with socioeconomic factors in measuring health determinants. Data from social media (Weibo) and mobile phones were analysed in two studies to better understand reactions of the general public to infectious diseases. One study, carried out by Fung et al. [10], revealed that Weibo (microblogs freely published through the Internet), proved useful to measure the Chinese people's reactions with respect to two different outbreaks, namely the 2012 MERSCoV outbreak [34] and the 2013 outbreak of H7N9 avian influenza A [35, 36]. Hence, the results suggest that Weibo could be a useful measure to raise public awareness by having health authorities release disease outbreak information via Weibo.

Kliner et al. [25] used a no-cost mobile phone agreement to send outpatient reminders for HIV testing and counselling in a pilot study in rural Swaziland, and found that mobile technology leading towards the concept of mobile health (mHealth) can be useful in many ways, initially by improving attendance at outpatient service clinics. Work stemming from Nigeria assessed household payments for TB with regard to incidence, determinants and policy implications for universal health coverage. The results suggest that current health costlowering strategies in Nigeria are not sufficient to prevent households from incurring catastrophic out-ofpocket payments for TB care [23]. To assess resources for the use of community-directed interventions (CDI), another study from Nigeria investigated delivery of health interventions in poorly-served urban communities in Ibadan. The research support the feasibility of using the CDI process in delivering health interventions in urban, poor environments and shows that resources for the strategy were potentially abound in the communities. 


\section{Visibility}

\section{Scope and transdisciplinary nature of the work}

Five articles reported on the estimation of disease burdens by measuring the impact of diseases at the local, national and global levels. With regard to rabies, one study collected all published articles and official documents on the disease in P.R. China in order to examine challenges to its elimination [13], while another discussed a global approach to rabies elimination emphasising the efforts needed to strengthen research and control activities, particularly in low- and middle-income countries [16].

Qian et al. [18] provided a concerted opinion on the tackling of clonorchiasis in P.R. China, while a review commented on the epidemiological status and characteristics of the disease at the global level and the aetiological relationship between cholangiocarcinoma and this infection [37]. The significance for clonorchiasis control and research priorities in P.R. China have been emphasised according to the pattern of recent epidemics and trend analyses in two papers [38, 39].

$\mathrm{TB}$ at the wildlife-livestock-human interface in Zambia was reviewed by Malama et al. [14], who called attention to the complex connection between the burden of animal Mycobacterium tuberculosis infections and its public health implications. The review pertaining to the epidemiology and interactions of HIV-1 and S. mansoni in sub-Saharan Africa indicates that $S$. mansoni infections can influence the replication of HIV-1 and deworming of HIV-positive individuals living in endemic areas, as it may impact on HIV-1 viral loads and $\mathrm{CD}^{+} \mathrm{T}$ lymphocyte counts [15].

Four research articles discuss the role of governments in disease endemic-countries and encourage cooperation in the field of zoonotic diseases [13, 15, 18, 26]. Another investigation highlights the collaboration between various governmental ministries as well as among medical and veterinary doctors by employing the "One World - One Health" concept [14].

\section{Innovative technology}

Surveillance-response approaches regarding tropical diseases in resource-constrained settings are increasingly applied in endemic countries with ongoing control and elimination programmes. Articles targeting such issues, taking into account environmental changes, social determinants and health systems considerations are in the limelight of the collection reviewed here. Indeed, among the 22 contributions, three have been viewed over 10,000 times within 4 months, 15 months and 22 months, respectively, after their publication $[10,24,26]$. The development of an innovative modelling approach entitled "Inferring the potential risks of H7N9 infection by spatiotemporally characterising bird migration and poultry distribution in eastern China", had the most views (14,904; accessed on 31
August 2015). Research on the social factors affecting the spread of infectious diseases entitled "Chinese social media reaction to the MERS-CoV and avian influenza A (H7N9) outbreaks" followed with 11,759 views.

In summary, it is fair to say that the importance of surveillance-response systems, representing the final crucial steps towards achieving effective control and elimination of communicable diseases, caused more attention than any other papers in this thematic issue of Infectious Diseases of Poverty.

In our analysis on the breadth and depth of the 22 contributions, we found that Chinese authors are becoming increasingly active with respect to control and elimination of tropical diseases. In concurrence with patterns recently published by Utzinger et al. [40] and Bergquist et al. [31, 40], the application of surveillanceresponse systems have developed into a necessary and sustainable part of the Chinese national disease control and elimination programmes [40-44].

\section{Conclusions}

Among the original research articles providing the core of this collection, seven research priorities emerged: (i) dynamic mapping of the transmission potential of various diseases; (ii) providing tools in near real-time capture of population dynamics; (iii) modelling based on minimum, essential databases; (iv) implementation of mHealth; (v) development of sensitive diagnostics; (vi) design of response packages tailored to different transmission settings and levels; and (vii) validation of surveillance and responses packages.

Through development and application of surveillanceresponse systems as part of the national diseases control and elimination programmes [40-44], P.R. China is becoming an important player in the area of the control and elimination of tropical diseases. It is in this connection essential that P.R. China, as one of the major contributors to the expansion of global health programmes, translates its experience into research and control of infectious diseases of poverty in places where these diseases are still widespread, including parts of P.R. China itself.

\section{Additional files}

Additional file 1: Multilingual abstracts in the six official working languages of the United Nations. (PDF $253 \mathrm{~kb}$ )

Additional file 2: List of the 22 articles in the thematic issue of Infectious Diseases of Poverty pertaining to surveillance-response systems that might lead to elimination of tropical diseases. (XLSX $158 \mathrm{~kb}$ )

Competing interests

The authors declared that they have no competing interests.

Authors' contributions

XZ conceived the study, collected and analysed the data and drafted the manuscript. PY, MT and RB conceived the project and provided technical 
support for data collection and analysis. JU and XNZ conceived the study and revised the manuscript. All authors read and approved the final manuscript.

\section{Acknowledgement}

This project was supported by the National S\&T Major Program (grant no. 2012ZX10004220), and the fourth round of Three-year Public Health Action Plan of Shanghai (2015-2017,No.GWIV-29).

\section{Author details}

'Department of Parasitology, Medical College of Soochow University, No. 199 Renai Road, Suzhou 215123, People's Republic of China. ${ }^{2}$ National Institute of Parasitic Diseases, Chinese Center for Disease Control and Prevention, 207 Rui Jin Er Road, Shanghai 200025, People's Republic of China. ${ }^{3}$ Swiss Tropical and Public Health Institute, P.O. Box, CH-4002 Basel, Switzerland. ${ }^{4}$ University of Basel, P.O. Box, CH-4003 Basel, Switzerland. IIngerod 407, S-454 94 Brastad, Sweden. ${ }^{6}$ Key Laboratory on Parasite and Vector Biology, Ministry of Health, WHO Collaborating Centre for Topical Diseases, 207 Rui Jin Er Road, Shanghai 200025, People's Republic of China.

\section{Received: 30 December 2015 Accepted: 5 May 2016 Published online: 14 May 2016}

\section{References}

1. TDR. Global Report for Research on Infectious Diseases of Poverty. Geneva: World Health Organization; 2012.

2. Bonds $\mathrm{MH}$, Keenan DC, Rohani P, Sachs JD. Poverty trap formed by the ecology of infectious diseases. Proc Biol Sci. 2010;277:1185-92.

3. Xia S, Allotey P, Reidpath DD, Yang P, Sheng HF, Zhou XN. Combating infectious diseases of poverty: a year on. Infect Dis Poverty. 2013;2:27.

4. Gyapong JO, Gyapong M, Yellu N, Anakwah K, Amofah G, Bockarie M, Adjei S: Integration of control of neglected tropical diseases into health-care systems: challenges and opportunities. Lancet. 2010;375:160-5.

5. $\quad$ Reeder JC. TDR: a time to live. Lancet. 2012;380:729-30.

6. Marais B, Crawford J, Iredell J, Ward M, Simpson S, Gilbert L, Griffiths P, Kamradt-Scott A, Colagiuri R, Jones C, Sorrell T: One world, one health: beyond the Millennium Development Goals. Lancet. 2012;380:805-6.

7. Zhou XN. Prioritizing research for "One health - One world". Infect Dis Poverty. 2012;1:1.

8. Zhou XN, Bergquist R, Tanner M. Elimination of tropical disease through surveillance and response. Infect Dis Poverty. 2013;2:1.

9. Bergquist $\mathrm{R}$, Whittaker M. Control of neglected tropical diseases in Asia Pacific: implications for health information priorities. Infect Dis Poverty. 2012;1:3.

10. Fung IC, Fu KW, Ying Y, Schaible B, Hao Y, Chan CH, Tse ZT: Chinese social media reaction to the MERS-CoV and avian influenza A (H7N9) outbreaks. Infect Dis Poverty. 2013;2:31.

11. Tambo E, Ai L, Zhou X, Chen JH, Hu W, Bergquist R, Guo JG, Utzinger J, Tanner M, Zhou XN: Surveillance-response systems: the key to elimination of tropical diseases. Infect Dis Poverty. 2014:3:17.

12. Liu DQ. Surveillance of antimalarial drug resistance in China in the 1980s1990s. Infect Dis Poverty. 2014;3:8.

13. Yin W, Dong J, Tu C, Edwards J, Guo F, Zhou H, Yu H, Vong S: Challenges and needs for China to eliminate rabies. Infect Dis Poverty. 2013;2:23.

14. Malama S, Muma JB, Godfroid J. A review of tuberculosis at the wildlifelivestock-human interface in Zambia. Infect Dis Poverty. 2013;2:13.

15. Mazigo HD, Nuwaha F, Wilson S, Kinung'hi SM, Morona D, Waihenya R, Heukelbach J, Dunne DW:. Epidemiology and interactions of human immunodeficiency virus - 1 and Schistosoma mansoni in sub-Saharan Africa. Infect Dis Poverty. 2013;2:2.

16. Zinsstag J. Towards a science of rabies elimination. Infect Dis Poverty. 2013;2:22.

17. Wiwanitkit V, Shi B, Xia S, Yang GJ, Zhou XN, Liu J. Research priorities in modeling the transmission risks of H7N9 bird flu. Infect Dis Poverty. 2013:2:17.

18. Qian MB, Chen YD, Yan F. Time to tackle clonorchiasis in China. Infect Dis Poverty. 2013;2:4.

19. Khan A, Hassan M, Imran M. Estimating the basic reproduction number for single-strain dengue fever epidemics. Infect Dis Poverty. 2014;3:12.

20. Meriki HD, Tufon KA, Afegenwi MH, Nyindem BA, Atanga PN, Anong DN, Cho-Ngwa F, Nkuo-Akenji T. Immuno-haematologic and virologic responses and predictors of virologic failure in HIV-1 infected adults on first-line antiretroviral therapy in Cameroon. Infect Dis Poverty. 2014;3:5.

21. Okamoto E. A mathematical model to predict the risk of hepatitis B infection through needle/syringe sharing in mass vaccination. Infect Dis Poverty. 2013;2:28.

22. Ajayi IO, Jegede AS, Falade CO, Sommerfeld J. Assessing resources for implementing a community directed intervention (CDI) strategy in delivering multiple health interventions in urban poor communities in southwestern Nigeria: a qualitative study. Infect Dis Poverty. 2013;2:25.

23. Ukwaja KN, Alobu I, Abimbola S, Hopewell PC. Household catastrophic payments for tuberculosis care in Nigeria: incidence, determinants, and policy implications for universal health coverage. Infect Dis Poverty. 2013;2:21.

24. Agomo CO, Oyibo WA. Factors associated with risk of malaria infection among pregnant women in Lagos, Nigeria. Infect Dis Poverty. 2013;2:19.

25. Kliner M, Knight A, Mamvura C, Wright J, Walley J. Using no-cost mobile phone reminders to improve attendance for HIV test results: a pilot study in rural Swaziland. Infect Dis Poverty. 2013;2:12.

26. Shi B, Xia S, Yang GJ, Zhou XN, Liu J. Inferring the potential risks of H7N9 infection by spatiotemporally characterizing bird migration and poultry distribution in eastern China. Infect Dis Poverty. 2013;2:8.

27. Amare H, Gelaw A, Anagaw B, Gelaw B. Smear positive pulmonary tuberculosis among diabetic patients at the Dessie referral hospital, northeast Ethiopia. Infect Dis Poverty. 2013;2:6.

28. Singh PK, Kushwaha S, Mohd S, Pathak M, Misra-Bhattacharya S. In vitro gene silencing of independent phosphoglycerate mutase (iPGM) in the filarial parasite Brugia malayi. Infect Dis Poverty. 2013;2:5.

29. Spellberg B, Taylor-Blake B. On the exoneration of Dr. William H. Stewart: debunking an urban legend. Infect Dis Poverty. 2013;2:3.

30. Ansa GA, Walley JD, Siddiqi K, Wei X. Assessing the impact of TB/HIV services integration on TB treatment outcomes and their relevance in TB/ HIV monitoring in Ghana. Infect Dis Poverty. 2012;1:13.

31. Bergquist R, Yang GJ, Knopp S, Utzinger J, Tanner M. Surveillance and response: tools and approaches for the elimination stage of neglected tropical diseases. Acta Trop. 2015;141:229-34.

32. National Institute of Parasitic Diseases: The First Forum on Surveillance Response System Leading to Tropical Diseases Elimination. Shanghai: National Institute of Parasitic Diseases, Shanghai, China; 2012. http://srs.ipd.org.cn:8080/srs/content/1st-forum-introduction.

33. Chen JH, Jung JW, Wang Y, Ha KS, Lu F, Lim CS, Takeo S, Tsuboi T, Han ET: Immunoproteomics profiling of blood stage Plasmodium vivax infection by high-throughput screening assays. J Proteome Res. 2010;9:6479-89.

34. Novel coronavirus infection in the United Kingdom. http://www.who.int/csr/ don/2012 09 23/en/index.html. 2012. (accessed on 23 Sept 2012).

35. Centers for Disease Control and Prevention: Emergence of avian influenza A(H7N9) virus causing severe human illness - China, February-April 2013. Morb Mortal Wkly Rep. 2013;62:366-71.

36. Three confirmed cases of human infections of H7N9 avian influenza in Shanghai and Anhui - Press release [in Chinese]. http://www.moh.gov.cn/ mohwsyjbgs/s3578/201303/44f25bd6bed14cf082512d8b6258fb3d.shtml. 2013. (accessed on 31 Mar 2016).

37. Qian MB, Chen YD, Liang S, Yang GJ, Zhou XN. The global epidemiology of clonorchiasis and its relation with cholangiocarcinoma. Infect Dis Poverty. 2012;1:4.

38. Qian MB. Clonorchiasis control: starting from awareness. Infect Dis Poverty. 2014:3:33.

39. Qian MB, Utzinger J, Keiser J, Zhou XN. Clonorchiasis. Lancet. 2016;387:800-10.

40. Utzinger J, Brattig NW, Leonardo L, Zhou XN, Bergquist R. Progress in research, control and elimination of helminth infections in Asia. Acta Trop. 2015;141:135-45.

41. Chen JH, Wang H, Chen JX, Bergquist R, Tanner M, Utzinger J, Zhou XN: Frontiers of parasitology research in the People's Republic of China: infection, diagnosis, protection and surveillance. Parasit Vectors. 2012;5:221.

42. Bergquist R. A century of schistosomiasis research. Acta Trop. 2008;108:65-8.

43. Utzinger J, Zhou XN, Chen MG, Bergquist R. Conquering schistosomiasis in China: the long march. Acta Trop. 2005;96:69-96.

44. Xu J, Xu JF, Li SZ, Zhang LJ, Wang Q, Zhu HH, Zhou XN: Integrated control programmes for schistosomiasis and other helminth infections in P.R. China. Acta Trop. 2015:141:332-41. 